\title{
Una legislación $100 \%$ libre de humo mejoró la salud de los trabajadores gastronómicos en una ciudad de Argentina
}

A $100 \%$ smoke-free law improved the health of gastronomic workers in a city of Argentina

Schoj y col. Tob Control 2010; 19:134-137.

\section{Objetivo}

Evaluar el impacto de la legislación de prohibición de fumar en espacios públicos cerrados (100\% libre de humo) en los síntomas de trabajadores de bares y restaurantes.

\section{Diseño}

Estudio de dos cortes transversales con evaluación anterior y posterior (estudio "antes-después") sin grupo de comparación.

\section{Lugar}

Fue realizado en la ciudad argentina de Neuquén.

\section{Participantes}

Una muestra de conveniencia de 80 trabajadores no fumadores de bares y restaurantes.

\section{Intervención}

Se realizó una encuesta de síntomas respiratorios y una espirometría basal un mes antes (Octubre 2007) y tres meses después (Marzo 2008) de la implementación de la nueva legislación 100\% libre de humo.

\section{Resultados}

Se observó una reducción en la exposición al humo ajeno, una disminución en los síntomas respiratorios (desde una prevalencia de $57,5 \%$ antes de la ley a $28,8 \%$ luego de la misma) y en los síntomas irritativos (desde $86,3 \%$ en octubre de 2007 a $37,5 \%$ en marzo de 2008) de los trabajadores gastronómicos luego de la implementación de la nueva legislación; así como un aumento significativo de la capacidad vital forzada (CVF). Todos estos cambios documentados fueron clínica y estadísticamente significativos. Los resultados se resumen en la tabla 1.

Tabla 1: exposición antes y después de la ley dentro y fuera del lugar de trabajo y síntomas respiratorios e irritativos en los trabajadores gastronómicos no fumadores.

\begin{tabular}{|c|c|c|c|c|c|}
\hline & & & $\begin{array}{l}\text { Antes de la ley } \\
\text { (2007) }\end{array}$ & $\begin{array}{l}\text { Luego de la ley } \\
\text { (2008) }\end{array}$ & p \\
\hline $\begin{array}{l}\text { Exposición en horas o } \\
\text { inter-cuartilo }\end{array}$ & iarias y rango & $\begin{array}{l}\text { En el lugar de trabajo } \\
\text { Fuera del trabajo }\end{array}$ & $\begin{array}{l}8 \text { (8 a 9) } \\
1(0 \text { a } 2)\end{array}$ & $\begin{array}{l}0(0 \text { a } 0) \\
0(0 \text { a } 2)\end{array}$ & $\begin{array}{l}<0,001 \\
0,0008\end{array}$ \\
\hline Porcentaje y número & Síntomas & Al menos un síntoma & $57,5 \%(46)$ & $28,7 \%(23)$ & $<0,001$ \\
\hline de individuos con & respiratorios & Tos & $20,2 \%(16)$ & $7,1 \%(5)$ & 0,039 \\
\hline síntomas & & Se despierta por tos & $31,6 \%(25)$ & $8,8 \%(7)$ & $<0,001$ \\
\hline & & Disnea por esfuerzo & $41,2 \%(33)$ & $16,2 \%(13)$ & $<0,001$ \\
\hline & & Disnea en reposo & $21,2 \%(17)$ & $5 \%(4)$ & 0,001 \\
\hline & Sintomas & Al menos un síntoma & $86,3 \%(69)$ & $37,5 \%(30)$ & $<0,001$ \\
\hline & irritativos & Ojos rojos & $58 \%(46)$ & $20 \%(16)$ & $<0,001$ \\
\hline & & Estornudos & $58,2 \%(46)$ & $26,2 \%(21)$ & $<0,001$ \\
\hline & & Picazón de garganta & $53,1 \%(42)$ & $17,7 \%(14)$ & $<0,001$ \\
\hline Parámetros de funció & pulmonara & Volumen espiratorio forzado en el primer segundo & $90 \%(11$ a 63$)$ & $90 \%$ (17 a 07$)$ & 0,8372 \\
\hline & & Capacidad vital forzada & $88 \%$ (11 a 55$)$ & $96 \%$ (12 a 88) & $<0,001$ \\
\hline
\end{tabular}

a: en porcentaje de la función esperada según edad, peso, talla y origen racial.

\section{Conclusiones}

Al igual que en otros estudios, la legislación $100 \%$ libre de humo mejoró los resultados de salud a corto plazo en nuestra muestra y debería implementarse en todo el país. Además, este estudio ha tenido gran importancia para promover la implementación de leyes $100 \%$ libres de humo en los lugares de trabajo como un derecho legítimo de los trabajadores gastronómicos y para reducir la aceptación social de las áreas para fumadores en bares y restaurantes.

Palabras claves: legislación, humo, trabajadores, impacto, salud.

Key words: laws, smoke, workers, impact, health.

Fuente de financiamiento/conflicto de interés de los autores: Fundación Bloomberg. Ningún conflicto de interés referido por los autores. 


\section{Comentario}

Al mes de febrero de 2011, el único país Sudamericano que no ha ratificado el Convenio marco para el control del tabaco es Argentina. Como hemos comentado en un reciente artículo de EVIDENCIA ${ }^{1}$, este tratado de salud pública de la Organización Mundial de la Salud (OMS) establece en su octavo artículo, que en los lugares cerrados de trabajo y cerrados de acceso público, el Estado debe aplicar medidas eficaces de protección a la exposición al humo ambiental de tabaco (HAT)2.

Desde hace décadas, la poderosa industria tabacalera mantiene una estrategia mundial, que en sus comienzos intentaba negar la evidencia del daño a la salud que provoca la exposición al humo ambiental de tabaco (HAT) y luego, sostener la falacia de que las áreas para fumar y no fumar en lugares cerrados eran suficientes para prevenir los daños ${ }^{3}$. Existe evidencia irrefutable de que la exposición al HAT aumenta el riesgo de padecer muchas enfermedades, algunas que aumentan la mortalidad como el enfisema, el cáncer pulmonar y los eventos coronarios agudos ${ }^{4}$. Tan importante es el impacto de la exposición al HAT en la salud, que luego de prohibir fumar en lugares públicos cerrados de un país, estado o ciudad, la incidencia de infartos agudos de miocardio en fumadores y no fumadores disminuye rápidamente, debido a la rápida reducción del riesgo cardiovascular ${ }^{5}$.

Las leyes de prohibición total de fumar en espacios cerrados (100\% libres de humo) son la principal amenaza para la industria tabacalera, ya que no sólo disminuyen el consumo de tabaco motivando a los fumadores a dejar o a fumar menos -los que no abandonan el tabaquismo, se estima que fuman aproximadamente $30 \%$ menos-, sino también la tolerancia social al uso de tabaco, ya que ponen en evidencia la necesidad de protegerse de un daño (el HAT). En ese sentido, vale destacar un estudio realizado por el Ministerio de Salud Nacional ${ }^{6}$, que analizó en forma comparada la Encuesta Mundial de Tabaquismo en Adolescentes realizada en 2000, 2003 y 2007 y que es representativa de los jóvenes de 13 a 15 años de los colegios secundarios del todo el país. Los resultados demuestran cambios significativos entre los años 2000 y 2007: en la Ciudad de Buenos Aires, en donde rige desde 2006 una legislación de ambientes libres de humo que disminuyó la tolerancia social al HAT significativamente, la prevalencia de tabaquismo en los adolescentes bajó de $25,3 \%$ a $18,5 \%$, la exposición al HAT bajó de $87 \%$ a $67 \%$ en lugares públicos y de $68 \%$ a $46 \%$ en el hogar, mientras que el apoyo de los jóvenes a las prohibi- ciones de fumar en lugares públicos aumentó de $70 \%$ a $88 \%$. Si bien la mejora en estos indicadores había comenzado antes de la ley y los factores socioeconómicos también influyen en estos resultados, es evidente que en las ciudades o provincias con legislación efectiva de ambientes libres de humo, la tendencia a una menor exposición al HAT y prevalencia de tabaquismo se hace mucho más marcada.

Los trabajadores gastronómicos, son uno de los "flancos débiles" del discurso de la industria tabacalera, ya que por el alto riesgo de enfermar y morir por el humo de segunda mano al que están expuestos durante todo el tiempo de trabajo en lugares sin prohibiciones, debilitan los argumentos que intentan frenar las prohibiciones totales de fumar en lugares cerrados de acceso público. El trabajo de Schoj y col. que hemos resumido, demuestra claramente que la exposición al HAT se reduce a cero y mejora la salud luego de la legislación $100 \%$ libre de humo, y que este tipo de legislación es fácilmente llevada a la práctica cuando existe voluntad política para hacerlo.

\section{Conclusiones de comentador}

Nuestra sociedad a avanzando lenta, pero persistentemente en la implementación de medidas de control del tabaco y el consumo, y la exposición al HAT está disminuyendo en la población general. Sin embargo, Argentina está muy atrasada en comparación con otros países, lo cual es esperable por la falta de medidas sanitarias adecuadas. En Argentina, el precio de los cigarrillos es muy bajo, no hay casi ninguna restricción a la publicidad del tabaco, se permite fumar en lugares públicos cerrados de numerosas regiones, no hay advertencias sanitarias adecuadas en los atados y no se garantiza la cobertura de los tratamientos para dejar de fumar. En síntesis, no existe hasta la fecha una estrategia nacional de control del tabaco que incluya una legislación nacional basada en medidas efectivas, como las provistas por el Convenio Marco de Control del Tabaco. La generación y difusión masiva de evidencia como la de Neuquén, es fundamental para contrarrestar la acción permanente de la industria tabacalera. Mientras no se aprueben e implementen leyes nacionales efectivas de control del tabaco, los avances locales que se están logrando por el impulso de la sociedad civil, son los que explican la lenta pero progresiva disminución de la prevalencia de tabaquismo en Argentina.

Javier Saimovici [ Servicio de Clínica Médica del Hospital Italiano de Buenos Aires y Departamento de Salud Pública de Instituto Universitario del Hospital Italiano. Javier.saimovici@ hiba.org.ar ]

Saimovici J. Una legislación 100\% libre de humo mejoró la salud de los trabajadores gastronómicos en una ciudad de Argentina. Evid Act Pract Ambul. 14(1).5-6. Ene-Mar 2011. Comentado de: Schoj V y col. The impact of a $100 \%$ smoke-free law on the health of gastronomic workers from the city of Neuquén, Argentina.Tob Control 2010; 19: 134-137.

\section{Referencias}

1. Chavez P y Schoj V. Convenio marco para el control del tabaco. Evid Act Pract Ambul. 13(4): 142-143. Oct-Nov.2010.

2. Organización Mundial de la Salud. Convenio Marco de la OMS para el Control del Tabaco. Disponible en URL: http://www.who.int/tobacco/framework/WHO_fctc_spanish.pdf (último acceso: 14/01/11).

3. Aguinaga Bialous, S y col. La rentabilidad a costa de la gente. Actividades de la industria tabacalera para comercializar cigarrillos en América Latina y el Caribe y minar la salud pública. Organización Panamericana de la Salud 2002

4. U.S. Department of Health and Human Services. The Health Consequences of Involuntary Exposure to Tobacco Smoke: A Report of the Surgeon General. U.S. Department 4. U.S. Department of Health and Human Services. The Health Consequences of Involuntary Exposure to Tobacco Smoke: A Report of the Surgeon General. U.S. Department
of Health and Human Services, Centers for Disease Control and Prevention, National Center for Chronic Disease Prevention and Health Promotion, Office on Smoking and

5. Pell J. Smoke-free Legislation and Hospitalizations for Acute Coronary Syndrome. N Engl J Med 2008;359:482-91.

6. Encuesta Mundial de Tabaquismo en Adolescentes en Argentina. Resultados de 2007 y comparación con encuestas previas. Ministerio de Salud Nacional 2009. Disponible en URL: http://www.msal.gov.ar/htm/site_tabaco/pdf/encuesta-tabaco-2008.pdf (último acceso: 14/3/2011) 